\title{
General note
}

The purpose of this workbook is to help you to learn how to work from engineering drawings of components which are to be machined and to produce part programs which incorporate the various commands and functions of a CNC system.

Most of the exercises in the workbook are practical and you will need to have access to a CNC system. This could be available either at a college or at your workplace.

Most CNC systems operate on similar principles, but the command syntax for positional data, tool calling and machining sequences varies. There are no definitive answers to these practical exercises. The way in which a programmer approaches a job depends on the jigs, fixtures and stock tooling that are available, as well as on the type of CNC machine tool that is to machine the part and on its programming functions. Many companies have a set procedure for their part programming, which may have been formulated over many years. This is particularly the case in the use of canned cycles for operations such as drilling holes, defining the tool change positions, methods of 'blocking out' (the rough cutting of the billet prior to finish machining) and the start and termination sequences of a CNC program.

\section{Safety note}

CNC machine tools move very fast in order to achieve the high production rates for which they are designed. It is therefore very important that, before using such a machine, you are completely familiar with the program and the machine slide/tool movements for the component that is to be machined. You should be given adequate supervision at all times. If you are at all in doubt, consult someone who is qualified to give advice.

An operator using

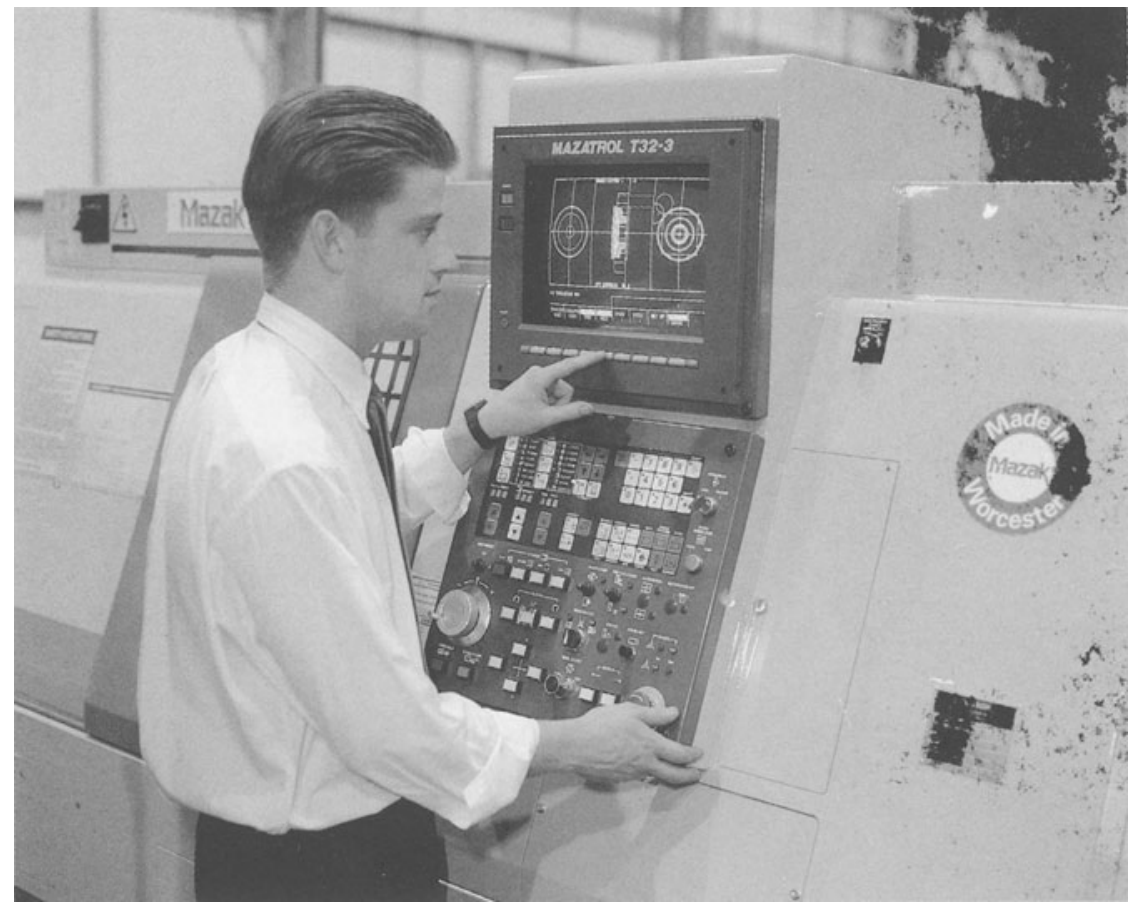

